\title{
Simulations of Laser Guide Star Adaptive Optics Systems for the European Extremely Large Telescope
}

\author{
R.M. Clare ${ }^{1, a}$ and M. Le Louarn ${ }^{1}$ \\ European Southern Observatory, Karl-Schwarzschild-Strasse 2, 85748 Garching, Germany
}

\begin{abstract}
End-to-end simulations of ground layer adaptive optics (GLAO) and laser tomography adaptive optics (LTAO) systems for the European extremely large telescope (EELT) are presented. This paper focuses on wavefront sensing issues; reconstruction is addressed in the paper by Bechet in these proceedings. For laser guide star based systems with Shack-Hartmann wavefront sensors without noise-optimal reconstruction, the simulations show that approximately 1.5 times as many photons are required for side launch of the laser as central launch to achieve the same performance in both LTAO and GLAO modes. The matched filter wavefont sensing algorithm provides better performance than correlation or center-of-gravity over the expected range of photon flux.
\end{abstract}

\section{Introduction}

The European extremely large telescope (EELT) is a $42 \mathrm{~m}$ primary mirror telescope currently in its design phase [1]. In order to fully utilize the unprecedented resolution of this telescope, adaptive optics (AO) will be employed to overcome the blurring effect of the atmosphere. The EELT will require a number of different AO systems [2], each designed to meet its instruments' science requirements. Single conjugate AO (SCAO) will use a natural guide star (NGS) to provide correction over a small field of view (FOV) with a single deformable mirror (DM). Due to the sky coverage constraints of NGS systems, multiple laser guide star (LGS) based systems are also required for the EELT. Ground layer AO (GLAO) will provide a modest correction over a large FOV, with a ground conjugated DM. Laser tomography AO (LTAO) will provide improved correction compared to GLAO, but over a much smaller field, again with a single DM. The multi conjugate AO (MCAO) system is similar to LTAO, except the correction will be applied with multiple DMs conjugated to different layers.

In this paper, we examine two LGS based AO systems: GLAO and LTAO, using ESO's end-to-end simulation tool Octopus [3]. In particular, we are interested in the preferred launch locations of the LGS, the required photon flux, the wavefront sensing algorithm, the detector sampling and FOV, the temporal sampling frequency as well as sensitivity to the atmospheric profile.

In Section 2, we define the GLAO and LTAO systems that we simulate. It is important to note that these are not baseline EELT designs, merely parametric studies on two types of LGS AO systems. End-to-end simulation results for these two LGS AO systems are presented in Section 3. Conclusions are drawn in Section 4, along with future areas of interest to improve the fidelity of the results.

\section{AO Systems Definition}

The atmospheric model used for both the GLAO and LTAO simulations in this paper is tabulated in Table 1. This is a 'median' profile, with a seeing of 0.8 arcsec along the line of sight. The atmospheric layers are scaled to an observing angle of 30 degrees from zenith. The corresponding isoplanatic angle is 2.0 arcsec, the coherence time $3 \mathrm{~ms}$, and the Fried parameter $12.9 \mathrm{~cm}$.

The AO system parameters common to GLAO and LTAO are shown in Table 2. These are the default parameters, although there is also a parametric analysis on some of those parameters. The $84 \times 84$ Shack-Hartmann configuration was chosen for the simulations as it is the one with the same sampling

\footnotetext{
a e-mail: rclare@eso.org
} the original work is properly cited. 
First conference on Adaptive Optics for Extremely Large Telescopes

Table 1. Atmospheric model used for GLAO and LTAO simulations.

\begin{tabular}{cccc}
\hline Layer & Height $(\mathrm{m})$ & $\%$ of $C_{N}^{2}$ & Wind speed $(\mathrm{m} / \mathrm{s})$ \\
\hline 1 & 47 & 53.28 & 15 \\
2 & 140 & 1.45 & 13 \\
3 & 281 & 3.5 & 14 \\
4 & 562 & 9.57 & 10 \\
5 & 1125 & 10.83 & 9 \\
6 & 2250 & 4.37 & 15 \\
7 & 4500 & 6.58 & 25 \\
8 & 9000 & 3.71 & 40 \\
9 & 18000 & 6.71 & 21 \\
\hline
\end{tabular}

Table 2. System parameters.

\begin{tabular}{ccc}
\hline Parameter & GLAO & LTAO \\
\hline WFS & $84 \times 84$ (Shack-Hartmann) & $84 \times 84$ (Shack-Hartmann) \\
Active actuators & 5400 & 5400 \\
Pixels / sub-aperture & $20 \times 20$ & $20 \times 20$ \\
Pixel Scale & 0.7 arcsec/pixel & 0.7 arcsec/pixel \\
Frame-rate & $500 \mathrm{~Hz}$ & $1 \mathrm{kHz}$ \\
Integrator Gain & 0.2 & 0.4 \\
Laser FWHM & $1.1 \mathrm{arcsec}$ & $1.1 \mathrm{arcsec}$ \\
\hline
\end{tabular}

as the specified adaptive mirror (M4) [2]. Actuators are considered active if they are controlled by the AO system. Inactive ones are present on the DM for structural reasons (support) but not directly controlled by the AO.

The wavefront reconstruction algorithm to generate the command matrix, $M$, is non tomographic. We make an interaction matrix with the LGS and NGS, $A$, and a single DM, and invert it with MAP regularization [4]:

$$
M=\left(A^{T} C_{n}^{-1} A+C_{\phi}^{-1}\right)^{-1} A^{T} C_{N}^{-1}
$$

where $C_{n}^{-1}$ is the noise covariance matrix and $C_{\phi}^{-1}$ is the Kolmogorov covariance matrix. The noise covariance matrix is calculated without taking into account the elongation of the LGS wavefront sensor (WFS) image in each subaperture. This means the consequent results presented for GLAO and LTAO are pessimistic as the reconstruction is non-optimal. Ref. [5] presents results with optimal noise weighting.

The number of pixels for the LGS WFS detectors, 20x20, is set by the required number in the LGS WFS detector trade study [6]. The read noise of 3e/pixel/readout is also the requirement as set out in [6]. The detector sampling is chosen to be $0.7 \mathrm{arcsec} /$ pixel. This value is chosen as the optimum of $\sim 1.5$ pixels/non-elongated FWHM found in [7] to balance between sampling errors and truncation. The temporal sampling frequency of the LGS WFS is $500 \mathrm{~Hz}$ for GLAO and $1 \mathrm{kHz}$ for LTAO. The number of photons/subaperture/frame refers to the number of photo-detection events on the detector. This number is per LGS, not total.

The launch location of the lasers is designated to be either side or central launch. In the case of central launch, all the lasers are projected from on-axis, i.e. at co-ordinates $(0,0)$ in the pupil plane. For side launch, four laser launch telescopes at $(+16.26,+16.26) \mathrm{m},(+16.26,-16.26) \mathrm{m},(-16.26,+16.26) \mathrm{m}$ and $(-16.26,-16.26) \mathrm{m}$ are used (i.e. $23.0 \mathrm{~m}$ off-axis so $2 \mathrm{~m}$ from edge of the primary). For GLAO, which has four LGS, this is one laser beacon per launch telescope. For LTAO, which has six LGS, two LGS are projected from each of two of the launch locations, and one LGS from each of the remaining two locations.

The LGS WFS spot size (short-exposure FWHM) is 1.1 arcsec in the non-elongated direction. This value comes from the median seeing of 0.8 arcsec and Eq. 6 of [8] for a launch telescope of 
$50 \mathrm{~cm}$ diameter. The sodium profile assumed is a Gaussian of FWHM of $11.4 \mathrm{~km}$, which is the median thickness in the Clemesha study [9], centred 90km above the telescope.

For LGS GLAO, the four LGS are equally spaced on a ring of diameter $5.0 \mathrm{arcmin}$. The NGS is on-axis and bright: 1e4 photons/subaperture/frame. The NGS WFS is a 1x1 Shack-Hartmann (i.e. only measures tip/tilt) and the detector (8x8 pixels) is noise free.

For LTAO, there is one LGS on-axis and the other 5 LGS are in a ring of radius 45 arcsec. The NGS is also on-axis and bright: 1000 photons/subaperture/frame. The NGS WFS for LTAO is a $3 \times 3$ Shack-Hartmann with a noise free $8 \times 8$ pixel detector.

Three WFS centroiding algorithms are explored in this paper. These are the traditional Centerof-Gravity (COG) algorithm, as well as the matched filter (MF) and correlation (CORR) algorithms. The exact form of the MF algorithm is the unconstrained version [10]. The exact form of the CORR algorithm is CORR with COG and thresholding on the correlation image [11]. The MF and CORR algorithms both require a reference image [7]. In the simulations, the reference images are initially calculated as Gaussians from the orientation of the subaperture relative to the laser launch telescope and the sodium thickness. The reference images are then calculated by accumulating the noisy images in each subaperture. The first 20 such frames are ignored while the loop is closing. After 60 frames (so 40 frames accumulated from time steps 20-60), the accumulated images have sufficient SNR to be used as the correlation images.

For GLAO, the performance metric used is the size of the pixel required for $50 \%$ Ensquared Energy (EE) in K band on-axis. For the GLAO simulations, each data point is run over 100 time steps $(200 \mathrm{~ms}$ at $500 \mathrm{~Hz}$ ). These relatively short time exposures are due to the slow speed of calculating the large FOVs required with elongated LGS WFS spots.

For LTAO, the performance metric is the long exposure (LE) Strehl (in K band) on-axis. This Strehl value includes all the fundamental AO terms: fitting, aliasing, tomography and temporal lag, not just the LGS WFS noise term. The LE Strehl is calculated from after the 30th time step. For LTAO simulations only 50 time steps are simulated so the LE Strehl is in fact only averaged over $20 \mathrm{~ms}$. This means tip-tilt is not taken into account, but should not be a limiting factor. This small sample size is a reason for the non-smooth nature of many of the curves presented in this paper.

\section{Simulation Results}

\subsection{GLAO Results}

Firstly, in Figure 1(a), the 50\% EE on-axis in $\mathrm{K}$ band is plotted versus the frame rate of the LGS WFS. For all points on this graph the flux per subaperture is kept constant at 100k photons/second (e.g. 200 photons/subaperture/frame at $500 \mathrm{~Hz}$ ). The exposure time is kept constant at 0.2 seconds for all points also (e.g. 100 time steps at $500 \mathrm{~Hz}$ ). The optimal sampling frequency is in the range $500-600 \mathrm{~Hz}$, and we use $500 \mathrm{~Hz}$ for the remainder of the GLAO simulations.

In Figure 1(b), the 50\% EE is plotted against the detected level of photons/subaperture/frame for LGS GLAO for side launch of the four LGS, central launch of the four LGS, and additionally for no elongation of the LGS spots. All three cases are with the default detector and the MF algorithm. Superimposed in Figure 1(b) is the 50\% EE due to the uncorrected atmosphere alone. Significantly more photons are required for side launch than central launch to obtain a given 50\% EE. For example, to achieve a $50 \% \mathrm{EE}$ of 0.30 arcsec, 95 photons are required for central launch, and 160 for side launch, a ratio of 1.7. For comparison, if there is no elongation a $50 \%$ EE of 0.30 arcsec can be achieved with 40 photons with the same detector. Of course, if there were no elongation, a smaller and lower noise detector could be used and fewer than 40 photons would be required. It is important to remember that all subapertures are weighted equally regardless of elongation here. The required photon flux of course depends on the level of performance (EE) required. However, it is clear that above 200 photons (central launch) or 300 photons (side launch) there is very little gain in increasing the LGS flux. Figure 1(b) shows that at low flux ( $<50$ photons) the EE is actually worse than the uncorrected atmosphere. This is partly because the system is optimized for higher fluxes. The gain, sampling frequency and the number of frames accumulated to make the matched filters were all chosen at high flux. Other parameters will be optimized in a future paper. 


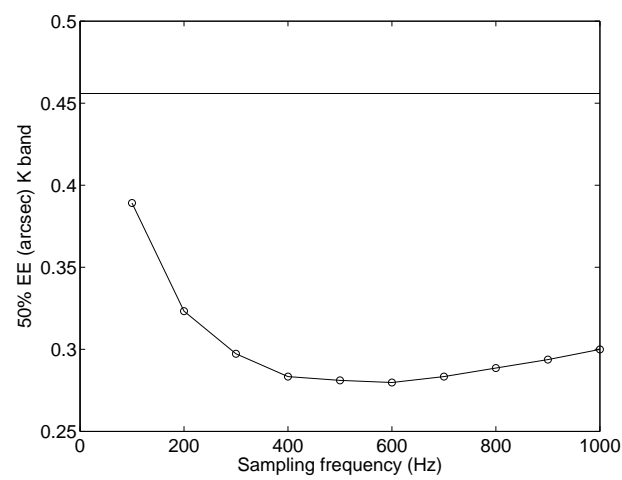

(a)

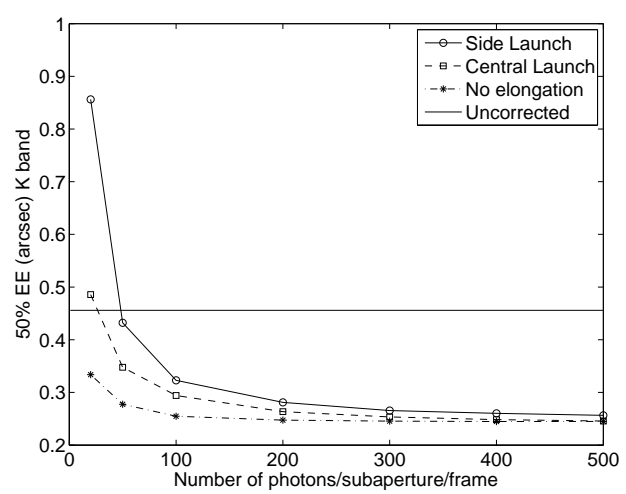

(b)

Fig. 1. The 50\% EE ( $\operatorname{arcsec}$ ) in K band for GLAO vs (a) the sampling frequency of the LGS WFS detectors for a constant flux of 100k photons/subaperture/s, and (b) the number of photons/subaperture/frame for side launch, central launch and for no elongation. In both cases the solid horizontal line is the $50 \% \mathrm{EE}$ for the uncorrected atmosphere.

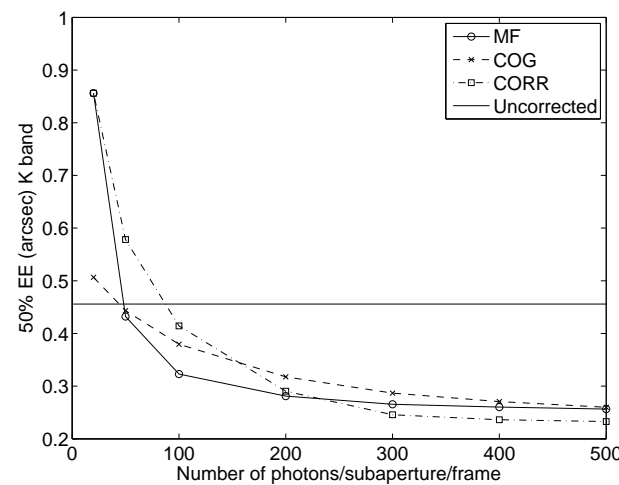

(a)

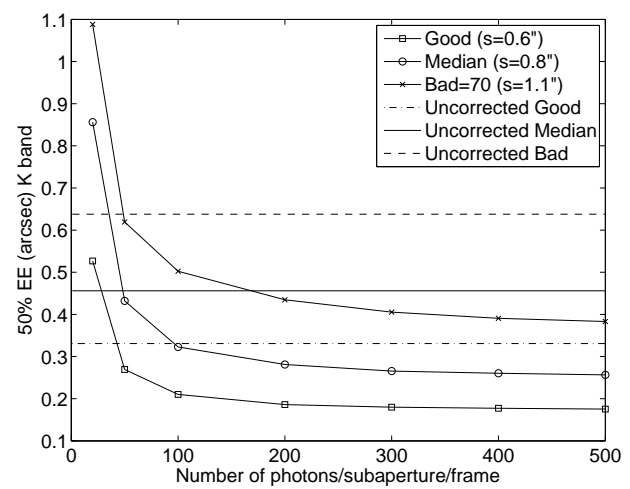

(b)

Fig. 2. The 50\% $\mathrm{EE}$ (arcsec) in $\mathrm{K}$ band for GLAO vs the number of photons/subaperture/frame for side launch for (a) different centroiding algorithms, and (b) different atmospheric profiles.

In Figure 2(a), the COG, MF and CORR centroiding algorithms are plotted versus number of photons/subaperture/frame for side launch of LGS GLAO. At low flux ( $<50$ photons), the COG algorithm performs the best, probably due to the poor MF and correlation reference images, which are only accumulated over 40 noisy frames. From 50-200 photons the MF gives the lowest EE, and above 200 photons the CORR algorithm yields the lowest $50 \%$ EE. One possible reason that the CORR algorithm does not do better at low flux is the sensitivity of the algorithm to the threshold on the COG of the correlation image. Thresholds of 40, 60 and $80 \%$ were tested at each signal level.

Three atmospheric profiles are investigated: good seeing ( $0.6 \operatorname{arcsec})$, median seeing ( 0.8 arcsec) and bad seeing (1.1 arcsec). The distribution of the strength of the layers is not significantly different (they all have $\sim 50 \%$ of energy at the lowest layer $-47 \mathrm{~m}$ ), and the windspeeds at each layer are identical for all 3 profiles. In Figure 2(b), the 50\% EE is plotted versus number photons/subaperture/frame for the good, median and bad atmospheric profiles. The performance of LGS GLAO is strongly dependent on the atmospheric profile assumed. In fact, no correction of the good profile provides better performance than with 500 photons with the bad profile. For all three profiles, GLAO correction can provide a factor $\sim 1.7$ improvement in the $50 \%$ EE. 


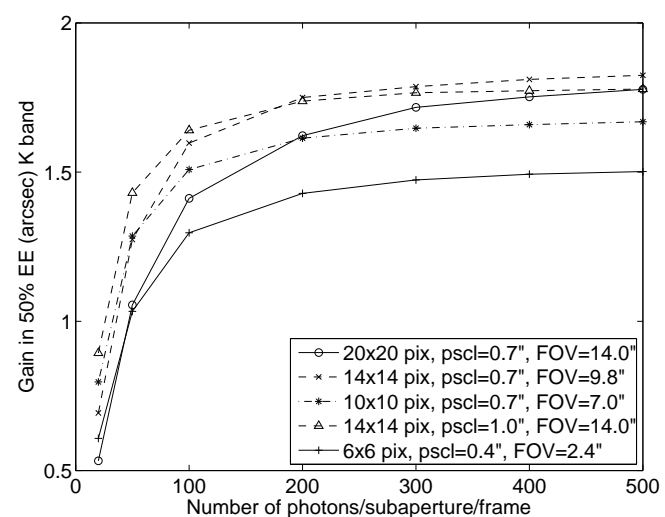

Fig. 3. Gain in 50\% EE compared to the uncorrected atmosphere versus number of photons/subaperture/frame for a number of combinations of FOV and pixel scale.

The FOV and sampling of the LGS WFS detector were optimized in Ref. [7] with a separate simulation tool. In particular, this optimization was done at high flux and for small jitters of the LGS WFS spots, more consistent with LTAO or MCAO than GLAO. It is not possible to do a full optimization again in the end-to-end simulator Octopus for GLAO, instead we run several cases around the assumed optimum. In Figure 3, the gain in 50\% EE compared to the uncorrected atmosphere for a number of FOV/pixel scales are plotted. It is necessary to plot the EE gain here, and not the absolute $50 \%$ EE, as the different FOV can have slightly different uncorrected 50\% EE. Figure 3 shows that the NGS GLAO detector (6x6 pixels, 0.4 arcsec/pixel) is by far the worst performed of the five combinations. At lower flux ( $<200$ photons), the coarser pixel scale (1.0 arcsec/pixel) gives the biggest gain, while at flux levels higher than this the combination of $14 \times 14$ pixels with $0.7 \mathrm{arcsec} /$ pixel is the best. However, the current default of $20 \times 20$ may be required to deal with wider Na profiles than the median $11.4 \mathrm{~km}$.

\subsection{LTAO Results}

Figure 4(a) compares the LE Strehl in K band on-axis of side and central launch of the six LGS of the LTAO system versus the subaperture signal level for the MF algorithm. For a given Strehl level, the side launch of the laser requires approximately 1.5 times more photons to reach this level than central launch of all the lasers. The maximum LTAO Strehl achieved is only 0.43 (even in the case of no noise and no elongation). This suggests the LTAO error budget is dominated not by LGS WFS noise, but by tomography and fitting errors. Figure 4(a) suggests that of the order of 300 photons are required for central launch of the laser; there is diminishing returns after this photon level.

In Figure 4(b) the Strehl is plotted for the CORR and MF algorithms with central launch. At flux levels below 300 photons/subaperture/frame the MF algorithm is significantly better than the CORR algorithm. This is possibly due to the threshold on the CORR algorithm not being optimal at low flux. The MF algorithm requires no such threshold optimization. At flux levels above 300 photons/subaperture/frame, the two algorithms perform similarly both achieving a Strehl of 0.43.

\section{Conclusions}

In this paper, we have studied a number of parameters influencing the performance of LGS based GLAO and LTAO systems for the EELT. We have shown that:

- Side launch of the lasers requires approximately 1.5 times as many photons as central launch to achieve either the same 50\% EE for GLAO or Strehl for LTAO.

- A significant improvement in 50\% EE (factor of 1.7 over the uncorrected atmosphere) for GLAO can be achieved with the order of 100-200 photons. 


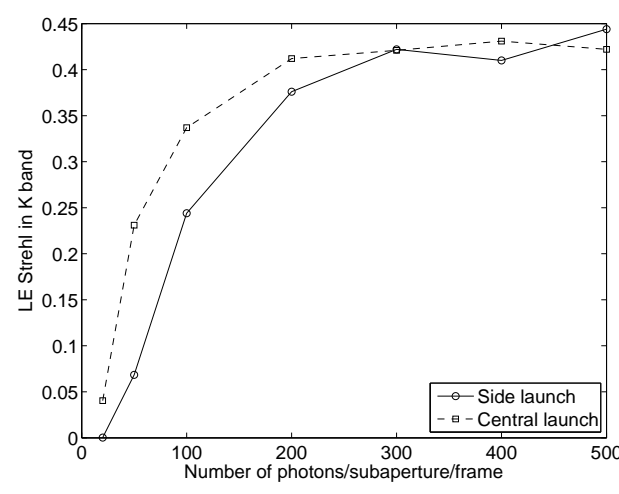

(a)

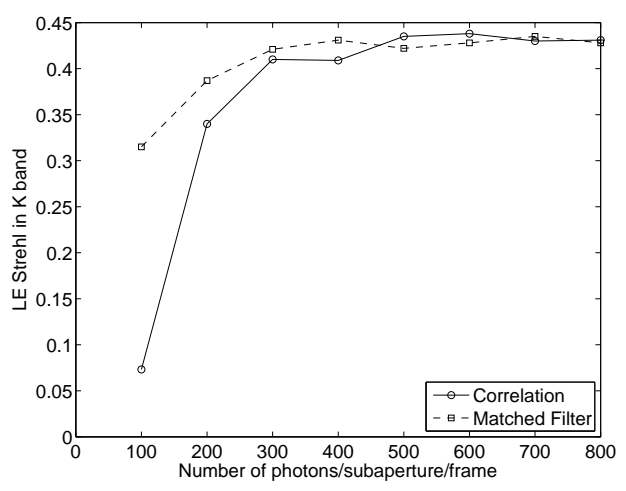

(b)

Fig. 4. The LE Strehl in K band for LTAO for (a) side and central launch with the MF algorithm, and (b) MF and CORR algorithms with central launch.

- The EE with LGS GLAO is strongly dependent on the atmospheric profile, however for all 3 atmospheric profiles tested, the EE can be improved by a factor of 1.7 with LGS GLAO.

- The matched filter is the preferred WFS algorithm.

- The optimal temporal sampling frequency for GLAO is approximately $500 \mathrm{~Hz}$.

The caveats for these conclusions are that the simulations are run over 50-100 time steps only, the sodium profile is assumed to be static and symmetric (Gaussian), and there are no static or quasi-static aberrations. Also, all the subapertures are weighted equally, regardless of the elongation.

In the future, we intend to study the effect of noise-weighting the subapertures as per Ref. [5], and the influence of the sodium profile on the wavefront sensing algorithms and control.

\section{References}

1. M. Kissler-Patig, "Overall science goals and top level AO requirements for E-ELT," in Adaptive Optics for Extremeley Large Telescopes, this volume.

2. N. Hubin, "Review of AO systems studied for the E-ELT," in Adaptive Optics for Extremeley Large Telescopes, this volume.

3. M. le Louarn, C. Verinaud, V. Korkiakoski, and E. Fedrigo, "Parallel simulation tools for AO on ELTS," in Advancements in Adaptive Optics, Proc. SPIE 5490 (2004).

4. N. F. Law et al., "Wavefront estimation at low light levels," Opt. Commun. 126, 19-24 (1996).

5. C. Béchet et al., "Closed-loop ground layer adaptive optics simulations with elongated spots : impact of modeled noise correlations," in Adaptive Optics for Extremeley Large Telescopes, this volume.

6. J. Kolb, "Detectors for LGS WF sensing on an ELT: characteristics, special features, limitations, technology," in Adaptive Optics for Extremeley Large Telescopes, this volume.

7. R. M. Clare, M. le Louarn, S. Oberti, and A. Garcia-Rissmann, "Adaptive optics simulations for the European Extremely Large Telescope," in Adaptive Optics Systems, Proc. SPIE 7015 (2008).

8. R. Holzlohner, D. Bonaccini Calia, and W. Hackenberg, "Physical optics modeling and optimization of laser guide star propagation," in Adaptive Optics Systems, Proc. SPIE 7015 (2008).

9. D. M. Simonich, B. R Clemesha, V. W. Kirchoff.,"The mesospheric sodium layer at 23degS: Nocturnal and seasonal variations", J. Geophys. Res. 84, 1543-1550 (1979)

10. L. Gilles and B. Ellerbroek, "Shack-Hartmann wavefront sensing with elongated sodium laser beacons: centroiding versus matched filtering," Appl. Opt. 45, 6568-6576 (2006).

11. L. A. Poyneer, "Scene-based Shack-Hartmann wave-front sensing: analysis and simulation," Appl. Opt. 42, 5807-5815 (2003). 